RHIC/AP/78

November 1995

\title{
Momentum collimation at Q9
}

\author{
S. Peggs, G.F. Dell
}

\section{Introduction}

Gold ions are prone to diffusion in transverse and longitudinal phase space, through Intra Beam Scattering (IBS). For example, the transverse emittance of a gold bunch is expected to increase to $40 \pi$ microns during a 10 hour store, while the longitudinal emittance rises to fill and overflow the bucket of the $197 \mathrm{MHz}$ RF system in which it is contained. If no remedial action is taken, gold ions that escape the RF bucket will streamline around the ring to give an approximately constant longitudinal background density. These particles will contribute to unwanted background in the experiments. If there is a sufficient number of them in the abort gap, they may cause magnet quenches when the beam is aborted - or dumped at the end of a fill.

This paper considers two simultaneous remedial actions:

1. Turn on the $28 \mathrm{MHz}$ accelerating RF system during storage. This creates 360 "macro-buckets", each one surrounding 7 storage buckets like peas in a pod. Gold ions that escape a storage bucket remain confined in the macro-bucket, where IBS diffusion rates are greatly reduced.

2. Remove gold ions that approach the macro-bucket separatrix with a momentum collimator installed next to a Q9 quadrupole, in a drift space where the horizontal dispersion is large. Only those Q9 locations where the beam and the collimator spray are moving away from the Interaction Point (IP), towards Q10, are sensible. Five such locations in each ring have vacant drifts that are, in principle, available for the installation of a momentum collimator. One location, downstream of the 6 o'clock IP, is already occupied by injection kicker modules. 


\section{Macro-buckets}

The small oscillation synchrotron tune is given by

$$
Q_{s 0}=\left(\frac{h \eta_{T}}{2 \pi} \cdot \frac{Z}{A} \cdot \frac{e V}{m_{0} c^{2} \gamma} \cdot \frac{1}{\beta^{2}}\right)^{\frac{1}{2}}
$$

where $V$ is the voltage of a single RF system with harmonic number $h, \eta_{T}$ is the slip factor, $Z$ and $A$ are the charge and nucleon counts of an ion with an average mass of $m_{0}$ per nucleon, and $\beta$ and $\gamma$ are relativistic factors. By direct analogy with transverse phase space nomenclature, the aspect ratio of a small bunch in longitudinal phase space $(z, \Delta p / p)$ is given by the longitudinal beta function $\beta_{z}$,

$$
\beta_{z} \equiv \frac{\sigma_{z}}{\sigma_{p} / p}=\frac{C}{2 \pi} \cdot \frac{\eta_{T}}{Q_{s 0}}
$$

where $C$ is the machine circumference and $\sigma_{z}$ is the rms bunch length. Finally, the maximum value of the off-momentum parameter $\delta=\Delta p / p$ on the separatrix is given by

$$
\widehat{\delta}_{\text {sep }}=\frac{\lambda}{\pi \beta_{z}}=\frac{2 Q_{s 0}}{h \eta_{T}}
$$

where $\lambda=C / h=3833.845 /(360 \times 7)=1.521 \mathrm{~m}$ is the RF wavelength of the storage system.

The case of interest is gold ions in storage at top energy, when $Z=79$, $A=197, m_{0}=0.93113 \mathrm{GeV} / \mathrm{c}^{2}, \gamma=108.395$, and $\eta_{T}=1.823 \times 10^{-3}$. In this case

$$
\begin{aligned}
& Q_{s 0}=4.174 \times 10^{-3}\left(\frac{V_{197}[\mathrm{MV}]}{6.0}\right)^{\frac{1}{2}} \\
& \widehat{\delta}_{\text {sep }}=1.818 \times 10^{-3}\left(\frac{6.0}{V_{197}[\mathrm{MV}]}\right)^{\frac{1}{2}}
\end{aligned}
$$

where the dependence on voltage is explicitly referred to $V_{197}=6.0 \mathrm{MV}$, the nominal value that is implicitly assumed from here on.

A concise description of the double RF system is given by a Hamiltonian that is parameterized by the storage RF quantities $Q_{s 0}$ and $\widehat{\delta}_{s e p}$

$$
H(p, \phi)=\left(2 \pi Q_{s 0}\right)^{2}\left[\frac{1}{2} p^{2}+(1-\cos (\phi))+7 v\left(1-\cos \left(\frac{\phi}{7}\right)\right)\right]
$$

One canonical coordinate is $\phi$, the $\mathrm{RF}$ phase of the $197 \mathrm{MHz}$ (storage) system, and the other is $p$, the scaled off-momentum parameter

$$
p=2\left(\delta / \widehat{\delta}_{s e p}\right)
$$


The equations of motion of this system are

$$
\begin{aligned}
\frac{d p}{d t} & \left.\equiv-\frac{\partial H}{\partial \phi}=-\left(2 \pi Q_{s 0}\right)\left[\sin (\phi)+v \sin \left(\frac{\phi}{7}\right)\right)\right] \\
\frac{d \phi}{d t} \equiv \frac{\partial H}{\partial p} & =\left(2 \pi Q_{s 0}\right) p
\end{aligned}
$$

where time, $t$, is measured in turns. Equation 8 confirms that $v$ is the scaled voltage of the $28 \mathrm{MHz}$ (acceleration) system

$$
v=\frac{V_{28}}{V_{197}} \leq \frac{0.8}{6.0}=0.133
$$

It has a maximum value of $v=0.133$ (when $V_{28}=0.8 \mathrm{MV}$ ) that may only be possible in pulsed, and not in $\mathrm{CW}$, operation [1]. It is reasonable and conservative to expect a CW value of $v=0.1$.

Inspection of the equations of motion in the limit of small $\phi$ shows that

$$
Q_{s 0}(v)=Q_{s 0}(0)\left(1+\frac{v}{7}\right)^{\frac{1}{2}}
$$

so that the synchrotron tune increases only slightly, by less than $2 \%$ at most. The momentum width of the macro-bucket separatrix is obtained by equating the value of the Hamiltonian $H(p, \phi)$ at its unstable fixed point, with the value at the widest part of the bucket

$$
H(0, \pm 7 \pi)=H(\widehat{p}, 0)
$$

and solving for $\widehat{p}$. Substitution of Eqns 6 and 7 reveals that

$$
\widehat{\delta}_{\text {sep }}(v)=\widehat{\delta}_{\text {sep }}(0)(1+7 v)^{\frac{3}{2}} \leq 2.526 \times 10^{-3}
$$

showing that the macro-bucket can be as much as $39 \%$ wider in momentum than a naked storage bucket. Figure 1 displays the longitudinal phase space behavior observed by the simulation code TEAPOT when $v=0.133$, independently confirming the quantitative predictions made above. 


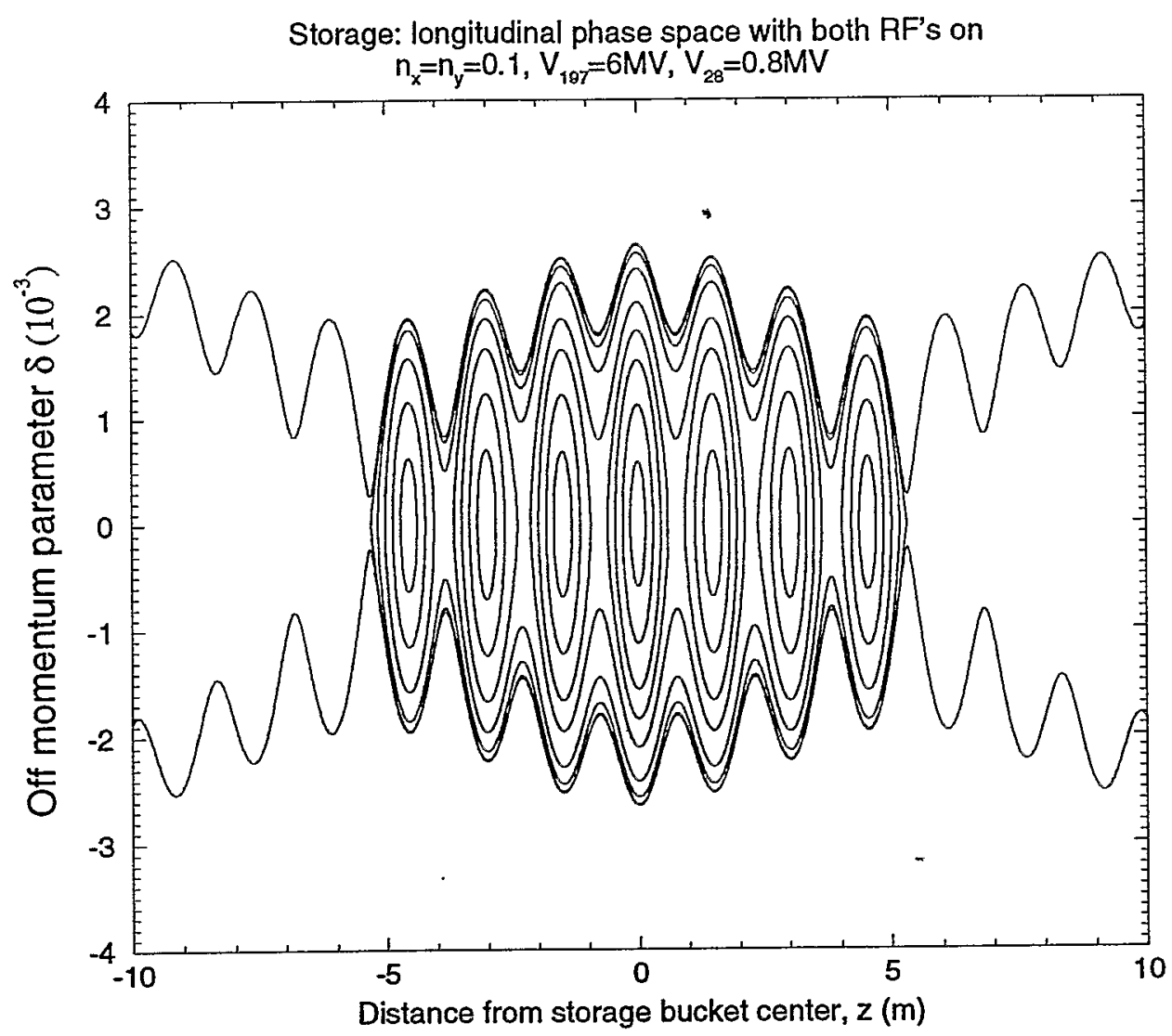

Figure 1: Longitudinal phase space, as observed with TEAPOT, when $V_{28}=$ $0.8 \mathrm{MV}$ and $V_{197}=6.0 \mathrm{MV}$. A "macro-bucket" of momentum width \pm 0.0025 surrounds 7 storage buckets, each approximately \pm 0.0018 wide. 


\section{Momentum collimation}

Suppose that a collimator is inserted from the radial outside of the beam to a (positive) displacement of $\widehat{x}$. A particle will just scrape the collimator, at some time in its history, if

$$
n_{x} \sigma_{x}+\eta_{x} \widehat{\delta}=\widehat{x}
$$

where $n_{x}$ is the horizontal betatron amplitude measured in units of the beam size $\sigma_{x}$, the horizontal dispersion is $\eta_{x}$, and $\widehat{\delta}$ is the maximum value of the offmomentum parameter. This equation can be rewritten to explicitly show how the maximum betatron amplitude depends on $\widehat{\delta}$,

$$
n_{x}(\widehat{\delta})=n_{x}(0)-\frac{\grave{\eta}_{x}}{\sigma_{x}} \widehat{\delta}
$$

where the parameter $n_{x}(0)$ is just the collimator offset measured in units of the beam size. If the goal of momentum collimation is to remove all particles with a momentum greater than some value $\widehat{\delta}_{\text {coll }}$, then

$$
n_{x}\left(\widehat{\delta}_{\text {coll }}\right)=0
$$

so that

$$
n_{x}(0)=\frac{\eta_{x}}{\sigma_{x}} \widehat{\delta}_{\text {coll }}
$$

For momentum collimation to be practical, the value of $n_{x}(0)$ must be large enough that only the inconsequential betatron tails of the on-momentum beam are scraped.

In the case of gold ions stored for 10 hours in RHIC, the normalized transverse emittance is taken to be $\epsilon=40 \pi \mu \mathrm{m}$, so that

$$
\sigma_{x}=\sqrt{\frac{\beta_{x} \epsilon}{6 \pi(\beta \gamma)}}=\sqrt{\frac{\beta_{x}[\mathrm{~m}]}{16.26}}[\mathrm{~mm}]
$$

yielding

$$
n_{x}(0)=4.03 \times 10^{3} \frac{\eta_{x}[\mathrm{~m}]}{\sqrt{\beta_{x}[\mathrm{~m}]}} \widehat{\delta}_{c o l l}
$$

This shows the important result that $\eta_{x} / \sqrt{\beta_{x}}$ is the fundamental optical "quality factor", of paramount importance when deciding where to place a momentum collimator. It is approximately constant in the dispersion matched FODO cells of the RHIC arcs, but falls to approximately zero in the dispersion suppressed interaction regions.

Table 1 shows the design optical parameters at potential momentum collimation locations, near Q9 quadrupoles downstream of interaction points with 


\begin{tabular}{|lcrrrr|}
\hline Name & s $[\mathrm{m}]$ & $\mu_{x} / 2 \pi$ & $\beta_{x}[\mathrm{~m}]$ & $\eta_{x}[\mathrm{~m}]$ & $\begin{array}{r}\eta_{x} / \sqrt{\beta_{x}} \\
{\left[\mathrm{~m}^{1 / 2}\right]}\end{array}$ \\
\hline & & & & & \\
clock6 & & & & & \\
q3o6 & 0.000 & 0.000 & 0.97 & 0.005 & 0.005 \\
q4ot6 & 35.830 & 0.246 & 506.95 & 0.470 & 0.021 \\
qda6 & 74.175 & 0.691 & 24.61 & 0.097 & 0.020 \\
INJECTION KICKER & 142.347 & 1.273 & 10.73 & 0.777 & 0.237 \\
erds & & & & & \\
ds & 150.974 & 1.354 & 29.38 & 1.390 & 0.256 \\
qf & 153.924 & 1.368 & 39.91 & 1.617 & 0.256 \\
& 157.163 & 1.379 & 48.83 & 1.795 & 0.257 \\
clock8 & & & & & \\
q3i8 & 639.445 & 5.239 & 0.97 & 0.005 & 0.005 \\
q4it8 & 675.275 & 5.485 & 1354.43 & -0.768 & -0.021 \\
qfa8 & 713.620 & 5.509 & 46.17 & -0.273 & -0.040 \\
F LOCATION $(1 \mathrm{~m})$ & 781.717 & 5.929 & 49.74 & 1.658 & 0.235 \\
erds & & & & & \\
ds & 790.339 & 5.973 & 19.68 & 1.167 & 0.263 \\
qd & 793.289 & 6.002 & 13.65 & 1.017 & 0.275 \\
& 796.523 & 6.047 & 10.59 & 0.919 & 0.282 \\
clock10 & & & & & \\
q3o10 & & & & & \\
q4ot10 & 1277.948 & 9.411 & 10.15 & -0.016 & -0.005 \\
qda10 & 1313.778 & 9.621 & 51.90 & -0.023 & -0.003 \\
D LOCATION (10 m) & 1420.295 & 10.651 & 10.61 & 0.822 & 0.252 \\
erds & & & & & \\
ds & 1428.923 & 10.732 & 29.51 & 1.452 & 0.267 \\
qf & 1431.872 & 10.746 & 40.19 & 1.685 & 0.266 \\
clock12 & 1435.112 & 10.757 & 49.27 & 1.866 & 0.266 \\
q3i12 & & & & & \\
q4it12 & 1917.393 & 14.581 & 9.90 & 0.017 & 0.005 \\
qfa12 & 1953.223 & 14.792 & 147.30 & 0.244 & 0.020 \\
F LOCATION (10 m) & 1991.568 & 14.925 & 17.85 & -0.067 & -0.016 \\
erds & 2059.666 & 15.365 & 48.69 & 1.597 & 0.229 \\
ds & 2068.288 & 15.411 & 19.53 & 1.154 & 0.261 \\
qd & 2071.237 & 15.439 & 13.68 & 1.020 & 0.276 \\
& 2074.471 & 15.484 & 10.78 & 0.941 & 0.287 \\
\hline
\end{tabular}

Table 1: Design optics near potential momentum collimator locations and other places of interest, in the Blue ring. Locations near F and D Q9 quads, and near $\beta^{*}=1$ and $10 \mathrm{~m}$ interaction points, are all roughly equivalent in $\eta_{x} / \beta_{x}^{1 / 2}$. 
Storage: Dependence of dynamic aperture on $\delta(\max )$ Set $n_{y}=0.1$ and vary $n_{x}$ (track for $24 K$ tums)

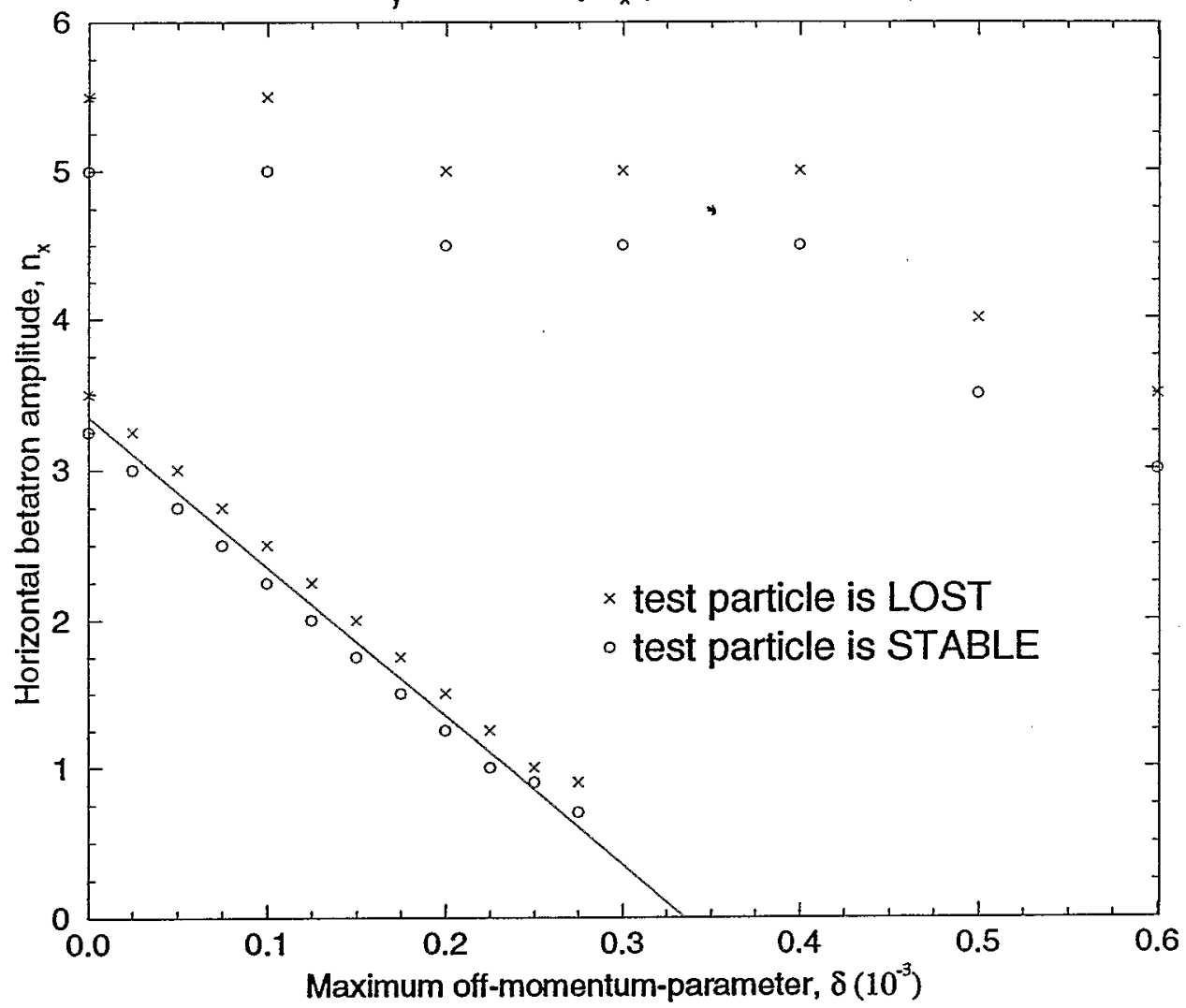

Figure 2: KHIC dynamic aperture after about 100 synchrotron periods, in a storage lattice with all errors turned on and all corrections applied. The lower solid line applies when a $4 \mathrm{~mm}$ aperture restriction is placed at Q9. The upper line shows the natural dynamic aperture, dominated by the triplet quadrupoles. In all cases the vertical betatron amplitude is $n_{y}=0.1$, while $V_{197}=6.0 \mathrm{MV}$ and $V_{28}=0.8 \mathrm{MV}$. 
low and high $\beta^{*}$ values $[2,3]$. All potential locations - whether $\mathrm{F}$ or $\mathrm{D}$, whether $\beta^{*}=1 \mathrm{~m}$ or $10 \mathrm{~m}$ - are roughly equivalent from an optical perspective. Taking a typical Q9 value of $\eta_{x} / \sqrt{\beta_{x}}=0.25\left[\mathrm{~m}^{1 / 2}\right]$, then

$$
n_{x}(0)=10^{3} \hat{\delta}_{\text {coll }}
$$

a fortuitously simple result. Finally, if momentum collimation is performed at the macro-bucket separatrix when $v=0.133$, then

$$
n_{x}(0)=10^{3} \widehat{\delta}_{\text {sep }}=2.5
$$

This shows that the collimation of on-momentum particles is NOT comfortably far off in the betatron tails, at least for gold ions with an emittance of $40 \pi$ microns. Figure 2 shows results from a TEAPOT simulation of momentum collimation, with a physical aperture of $4 \mathrm{~mm}$ asserted at the Q9 quadrupole downstream of the $80^{\prime}$ 'clock IP. These results confirm the simple picture given above, although there are signs that nonlinear and chromatic effects are not completely negligible.

\section{Conclusions}

Macro-buckets created by turning on the $28 \mathrm{MHz}$ RF system may be used to trap gold ions that diffuse out of the storage buckets.

A momentum scraper at any one of five downstream Q9 locations in each ring may be used to remove gold ions that approach the macro-bucket separatrix. Unfortunately, these locations require the added expense of warm-to-cold transitions.

A momentum collimator near Q9 reaches uncomfortably close - about $2.5 \sigma_{x}$ to the betatron core of on-momentum gold ions, when the transverse emittance is at its largest, $\epsilon=40 \pi$ microns. A modest improvement in optical or $\mathrm{RF}$ parameters - raising $\widehat{\delta}_{\text {sep }}$, raising $\eta_{x}$, or lowering $\beta_{x}$ - would move Q9 momentum collimation from "satisfactory" to "solid" performance.

A Q9 momentum collimator would NOT be efficient as a secondary betatron. collimator, in conjunction with a primary betatron collimator just upstream, between Q3 and Q4. Even though the betatron phase advance is near optimum, the completely different values of $\eta / \sqrt{\beta_{x}}$ at the two locations makes the combination very ill-matched [4]. 


\section{References}

[1] M. Brennan, private communication.

[2] The design optics data were taken from the file /rap/Holy_Lattice/Blue/twiss_store.asc .

[3] S. Tepikian, "Twiss Functions and Beam Sizes of the RHIC Insertion", RHIC/AP/25, 1994.

[4] M. Seidel, "The Proton Collimation System of HERA", PhD thesis, DESY 94-103, 1994. 